\title{
Perfusion assessed by real-time contrast echocardiography correlates with clinical and echocardiographic parameters in patients with first STEMI treated with $\mathrm{PCI}$ - 6-month follow-up
}

\author{
Anna Tomaszuk-Kazberuk ${ }^{1}$, Bozena Sobkowicz ${ }^{1,2}$, Slawomir Dobrzycki ${ }^{2,3}$, Anna Lewczuk ${ }^{1,2}$, \\ Włodzimierz Musial ${ }^{1,2}$
}

1Department of Cardiology, Medical University of Bialystok, Poland
2University Hospital of Bialystok, Poland
${ }^{3}$ Department of Invasive Cardiology, Medical University of Bialystok, Poland

Submitted: 21 December 2008

Accepted: 29 March 2009

Arch Med Sci 2010; 6, 2: 176-182

DOI: 10.5114 /aoms.2010.13890

Copyright (C) 2010 Termedia \& Banach

\section{Abstract}

Introduction: Angiographic flow in an epicardial artery does not define perfusion at a microvascular level in patients with acute myocardial infarction (AMI). The aim of the study was assessing microvascular reperfusion by myocardial contrast echocardiography (MCE) and left ventricular (LV) functional recovery by echocardiographic methods in patients treated with primary PTCA.

Material and methods: 100 consecutive patients with first ST-elevation AMI with single vessel disease treated successfully with primary $\mathrm{PCI}$ were enrolled. Regional contrast score index (RCSI), ejection fraction (EF), wall motion score index (WMSI), and end-systolic and end-diastolic volume (ESV, EDV) were evaluated during hospitalization and at 6-month follow-up. The patients were divided into 2 groups according to the absence (Group 1) or presence (Group 2) of perfusion defects on MCE.

Results: Group 1 had lower WMSI ( $p=0.0009)$, higher EF than Group 2 (44.7 and $55.9 \%$ respectively, $p=0.000067)$, and lower ESV $(66.0$ and $52.6 \mathrm{ml}$ respectively, $p=0.003185)$. In Group 1 LVEF increased significantly on 6-month follow-up $(p=0.026)$, while in Group 2 it decreased $(p=0.0175)$. Both EDV and ESV were significantly lower in Group $1(p=0.0106$ and $p=0.002882$, respectively). There was a correlation between the presence of perfusion defects in the initial contrast echo and unfavourable change in ejection fraction during the follow-up (ANOVA for repeated measures, $F[1.91]=5.85, p=0.0175$ ). The combined clinical end-point (death and reinfarction) was significantly lower in patients without perfusion defect $(p=0.039)$.

Conclusions: Myocardial contrast echocardiography results correlated with clinical outcome and recovery of systolic left ventricular function at 6-month follow-up.

Key words: myocardial perfusion, contrast echocardiography, left ventricular function, survival after myocardial infarction.

\section{Introduction}

Angiographic flow in an epicardial artery does not accurately define perfusion at a microvascular level [1]. A lack of myocardial reperfusion in spite of restored flow, the "no-reflow" phenomenon, was initially revealed by myocardial contrast echocardiography and confirmed by other imaging
Corresponding author:

Anna Tomaszuk-Kazberuk, $\mathrm{PhD}$

Department of Cardiology

Medical University

of Bialystok

E-mail: walkaz@mp.pl 
techniques such as positron emission tomography $[2,3]$. In AMI a lack of myocardial perfusion despite recanalization of the infarct-related artery (IRA) is associated with an increased rate of left ventricular dysfunction, complications and impaired survival [4-7].

Therefore there is a need to apply techniques assessing flow at a tissue level at bedside. Myocardial contrast echocardiography (MCE) in AMI provides information regarding the adequacy of myocardial tissue perfusion as well as the spatial extent of microvascular obstruction.

There are limited data showing perfusion assessed by contrast echocardiography with echocardiographic contractility parameters in patients with first AMI successfully treated with angioplasty on follow-up.

The purpose of this study was to assess microvascular reperfusion by MCE, LV functional recovery by echocardiographic methods and clinical outcome in patients with first AMI successfully treated with primary PTCA.

\section{Material and methods}

\section{Study population}

Hundred consecutive patients with a first AMI admitted within $12 \mathrm{~h}$ of onset of chest pain, and treated with primary $\mathrm{PCl}$, were enrolled in our study. Only patients with single vessel disease were included in the present study. Exclusion criteria were: 1) cardiogenic shock at the time of MCE, 2) unsuccessful angioplasty, TIMI flow post $\mathrm{PCI}<3$ and residual lesion $>30 \%, 3$ ) clinical history of congestive heart failure or valve disease, 4) pregnancy or breastfeeding, 5) life-threatening condition other than $\mathrm{AMI}, 6)$ inadequate thoracic window for echocardiography. No upper age limit was defined.

The study complies with the declaration of Helsinki. The study protocol was approved by the local ethics committee and informed consent was obtained from all subjects participating in the study. Clinical characteristics are shown in Table I.

Almost all patients received aspirin, $\beta$-blockers, ACE inhibitors, and statins from the first day of treatment. In case of stent implantation $(90 \%$ of patients) clopidogrel was administered.

The patients were divided into 2 groups according to absence (Group 1) or presence (Group 2) of perfusion disturbances on MCE.

\section{Echocardiographic analysis}

All studies were performed using the Philips Ultrasound System Sonos 5500 equipped for harmonic imaging and $3.6 \mathrm{MHz}$ transducer. Instrument setting for myocardial contrast echocardiography was optimized in order to have maximum sensitivity and ideal conditions for visual myocardial contrast detection. The recommended dynamic range was in the medium or mid range (45-55 dB); focal zone depth was set at approximately $2 / 3$ of the image; gain was adjusted so that myocardial tissue speckle details could be seen on baseline images (this resulted in homogeneous grey backscatter throughout the entire wall of the left ventricle). Mechanical index was set between 0.3 and 0.9. Thereafter, all settings were kept constant during the acquisition of images. The dysfunctional area was visualized using harmonic imaging in either the 4- or 2-chamber view. ECG triggering was done in late end-systole at every third heart beat with image acquisition at the T-wave of the ECG signal.

Table I. Clinical characteristics of patients

\begin{tabular}{|lccc|}
\hline $\begin{array}{l}\text { Evaluated } \\
\text { parameters }\end{array}$ & $\begin{array}{c}\text { Patients without perfusion } \\
\text { defect } n=53\end{array}$ & $\begin{array}{c}\text { Patients with perfusion } \\
\text { defect } n=47\end{array}$ & Value of $p$ \\
\hline Mean age [years] & $58.3(\mathrm{SD} \pm 11.33)$ & $60.8(\mathrm{SD} \pm 12.82)$ & 0.31 \\
\hline Male [\%] & 56.9 & 58.2 & 0.68 \\
\hline Hypertension [\%] & 64.2 & 65.2 & 0.91 \\
\hline Diabetes mellitus [\%] & 37.7 & 26.1 & 0.22 \\
\hline Dyslipidaemia [\%] & 60.4 & 63.1 & 0.79 \\
\hline Smoking [\%] & 17.3 & 21.7 & 0.58 \\
\hline CK-MB (max. value IU/l) & $197.8(\mathrm{SD} \pm 129.2)$ & $244.2(\mathrm{SD} \pm 161.9)$ & 0.13 \\
\hline Patients with anterior MI [\%] & 24.5 & 63.6 & 0.000014 \\
\hline Time from pain onset to balloon & $270.7(\mathrm{SD} \pm 171.4)$ & $288.6(\mathrm{SD} \pm 223.5)$ & 0.68 \\
\hline TIMI before PCl & $0.8(\mathrm{SD} \pm 1.2)$ & $0.9(\mathrm{SD} \pm 1.2)$ & 0.93 \\
\hline Number of stents & $1.1(\mathrm{SD} \pm 0.4)$ & $1.0(\mathrm{SD} \pm 0.4)$ & 0.22 \\
\hline Ilb/IIla - tirofiban [\%] & $16.9(n=9)$ & $7.5(n=4)$ & 0.019 \\
\hline
\end{tabular}


Baseline regional wall motion score index (WMSI) and regional contrast score index (RCSI) were calculated at least $48 \mathrm{~h}$ after $\mathrm{PCl}$. Optison ${ }^{\circledR}$ contrast agent was administered via a peripheral vein. Myocardial contrast echocardiography was performed using a modality of subsequent data acquisition of triggered end-systolic images. The criterion for good perfusion on MCE was defined as homogeneous enhancement in $50 \%$ of wall thickness in each segment.

Echocardiographic images were digitally stored in a cine loop format for off-line analysis by 2 experienced observers. Discrepancies were resolved by consensus. Baseline fundamental imaging, including 2 parasternal (long and short axis) views, were used to evaluate baseline regional and global wall motion score indexes using the 16-segment model prior to myocardial contrast echocardiography [8]. For each wall segment, motion was scored as 1 (normal), 2 (hypokinetic), 3 (akinetic), or 4 (dyskinetic). Left ventricular ejection fraction (LVEF) and wall motion score index (WMSI) were obtained for all echo scans. Wall motion score index was obtained by dividing the sum of the segment scores by the number of segments scored.

Left ventricular ejection fraction was derived using the modified bi-plane Simpson's method, from orthogonal apical long-axis projections (4-chamber view and 2-chamber view). All measurements were derived in blinded fashion by 2 experienced operators. The 3 mean measurements of the best visualized cardiac cycles were calculated for each echo study.

\section{Application of contrast agent}

After pre-injection recordings, the ultrasound contrast agent Optison ${ }^{\circledR}$ was injected intravenously. This contrast agent consists of perfluorocarbon filled microbubbles with a median diameter of 3-4 $\mu \mathrm{m}$ (Amersham, USA). A dose of $1 \mathrm{ml}$ of contrast agent followed by $10 \mathrm{ml}$ of saline was injected. This was chosen following the results of pilot studies. One bolus injection was administered for each echocardiographic view [9]. At least 5 beats prior to and 30 beats after the injection were recorded for each view.

\section{Definitions}

Myocardial perfusion of each segment was assessed using the following grading: poor or no opacification, partial opacification, adequate opacification or artefacts. Adequate opacification was scored when the segment showed homogeneous opacification in at least one view. Partial opacification was scored as inadequate myocardial enhancement in any view relative to adjacent segments with adequate opacification. Lack of opacification was scored as low myocardial enhancement. Artefacts were differentiated from perfusion defects prior to final analysis. Based on MCE results, patients were categorized according to the presence or absence of a myocardial perfusion defect in at least one segment in the dysfunctional area.

The presence of myocardial perfusion was defined as adequate opacification in that segment. Areas of no or poor myocardial opacification were regarded as myocardial perfusion defects.

RCSI was calculated by dividing the sum of perfusion assessment of every asynergic segment by the number of evaluated segments. For each segment good myocardial perfusion was scored as 1. Segments with partial, poor or no opacification were scored as 0. Range of RCSI was 0-1 [10]. In cases of normal perfusion, RCSI was 1 .

\section{Primary coronary intervention}

In all patients coronary angiography and intervention were performed within $12 \mathrm{~h}$ of the onset of symptoms. Ninety patients underwent primary stent implantation. Arterial access was by the right femoral artery. Selective coronary arteriography was performed using standard techniques [11]. The infarctrelated artery (IRA) was identified based on coronary anatomy, regional LV dysfunction and ECG changes.

The $\mathrm{PCl}$ procedure was considered successful when the residual stenosis was $<30 \%$, in the absence of dissection. The IRA was analysed both before and after primary coronary angioplasty to assess the residual stenosis. Contrast flow through the epicardial vessel was graded using the standard TIMI trial flow scale of 0 to 3 . All angiograms were analysed by 2 observers blinded to clinical and echocardiography results. In the case of disagreement, a third observer reviewed the film and discrepancies were resolved by consensus.

Pharmacological treatment in the catheterization laboratory included intravenous heparin at the beginning of the procedure. Thirteen patients were treated with the IIb/IIla inhibitor (tirofiban) at the discretion of the $\mathrm{PCl}$ operator.

\section{Statistical analysis}

Continuous variables were expressed as mean \pm standard deviation and compared by means of 2-tailed unpaired $t$-test (for parametric variables) and Mann-Whitney U-test (for non-parametric variables). Discrete variables were expressed as percentages of the study population and compared by the $\chi^{2}$ test or Fisher's exact test. To assess the correlation between the number of segments with perfusion defect and clinical, laboratory and echocardiographic variables, Spearman test was used. Perfusion defects 
assessed by echocardiography were compared in various groups of patients established according to tissue perfusion on angiography using the analysis of variance (ANOVA) with post-hoc Tukey test. Analysis of EF, EDV and ESV during follow-up was also performed using ANOVA for repeated measures. For assessment of predictors of reperfusion a multivariate analysis was used. A value of $p<0.05$ was considered statistically significant. Statistical analyses were performed with Statistica 6.0 for Windows (2000).

\section{Results}

A total of 100 consecutive patients were included, of whom all were available for echocardiographic analysis. There were no significant differences between groups with respect to age, sex, heart rate, arterial pressure and pharmacological treatment as well as mean painto-balloon time (Table I).

Group 1 consisted of 53 patients without perfusion defect on MCE, while in 47 patients (Group 2) segments with perfusion disturbances were found. Mean RCSI in Groups 1 and 2 was $1 \pm 0.0$ and $0.56 \pm 0.4$, respectively $(p=0.0064)$.

From 1600 segments in all enrolled patients we analysed 717 asynergic segments. Among the 717 segments assessed by MCE, 168 (22\%) revealed partial or total lack of perfusion. Sixteen segments had inadequate MCE images due to difficulties with imaging of the medial and basal segments of the lateral wall. These 16 segments were not included in the analysis. Medial and basilar segments of lateral wall were assessed only in lateral and anterolateral infarctions.

\section{Relationship between myocardial perfusion defects assessed by myocardial contrast echocardiography and clinical and laboratory parameters}

In Group 1 the infarct-related artery was the left anterior descending coronary artery in 16 patients (30.1\%), the right coronary artery in 31 patients (58.5\%), and the circumflex coronary artery in 6 patients (11.4\%). In Group 2 the infarct-related artery was the left anterior descending coronary artery in 29 (63.0\%), the right coronary artery in 12 (26.1\%), and the circumflex coronary artery in 5 (10.9\%).

More anterior infarcts were found and consequently the infarct-related artery was more often the left anterior descending in patients with a perfusion defect (Group 2). Out of 53 patients from Group 1 only 13 had anterior myocardial infarction, whereas in Group 2 there were 29 anterior myocardial infarctions $(p=0.00014)$.

Patients with anterior AMI had significantly more segments with perfusion defect evaluated with
MCE than patients with inferior myocardial infarction ( $p=0.0001)$.

Patients from the group with perfusion defect had more segments with impaired contractility $(p=0.0001)$ and higher WMSI $(p=0.0009)$. The mean number of segments with contractility disturbances in Group 1 was 5 and in Group 2 was 9. Regional WMSI in Group 1 and Group 2 was 1.4 and 1.6 , respectively $(p=0.0009)$.

Group 1 had higher ejection fraction (EF biplane) than Group 2 (44.7 \pm 8.2 and $55.9 \pm 7.4 \%$ respectively, $p=0.000067)$, lower ESV (66.0 \pm 6.3 and $52.6 \pm 7.2 \mathrm{ml}$ respectively, $p=0.003185)$ and lower WMSI (1.47 \pm 0.3 and $1.60 \pm 0.4$ respectively, $p=0.0009$ ) (Table III). There were no differences between the groups in EDV (118.3 and $110.5 \mathrm{ml}$, respectively, $p=0.29$ ).

In the multivariate analysis, absence of anterior myocardial infarction $(p=0.0017)$, high $\mathrm{EF}$ on echocardiography $(B=-0.31, p=0.006)$ and use of Ilb/Illa blockers $(B=-0.31, p=0.004)$ were the only predictors of reperfusion on MCE.

Only 13 patients from the whole study group received tirofiban, $16.9 \%$ of patients from the group without perfusion defect and $7.5 \%$ from the group with perfusion defect. The patients treated with tirofiban had significantly lower RCSI than those who did not receive tirofiban $(p=0.003)$.

Smoking was more frequent in patients with perfusion defect on MCE but the difference was at the limit of significance $(p=0.053)$. In multivariate analysis smoking $(B=0.2, p=0.053)$ and the highest value of CK-MB activity within 2 days after admission to the hospital $(B=0.21, p=0.037)$ were predictors of perfusion defect on MCE. Peak CK-MB correlated well with WMSI $(p=0.037)$ and with RCSI in Group 2 ( $p=0.037)$.

During in-hospital stay we did not observe any serious cardiovascular events, which is not surprising when one considers the 1-vessel coronary disease population.

\section{Six-month clinical follow-up}

Ten patients were unavailable for follow-up: 2 from Group 1 and 8 from Group 2. In Group 1, LVEF increased significantly from baseline $52.1 \%$ on admission to $55.9 \%$ on 6 -month follow-up ( $p=0.026)$, while in Group 2, it decreased from $45 \%$ on admission to $43 \%$. The difference between the groups in LVEF was statistically significant ( $p=0.0175)$. Both EDV and ESV were significantly lower in Group $1(113.5$ and $137.8 \mathrm{ml}$, respectively, $p=0.0106 ; 59.0$ and $82.0 \mathrm{ml}$ respectively, $p=0.002882$ ). There was a statistically significant correlation between the presence of perfusion defects in the initial contrast echo and the change in ejection fraction during the follow-up (ANOVA for repeated measures, $p=0.0175$ ) (Figure 1). 


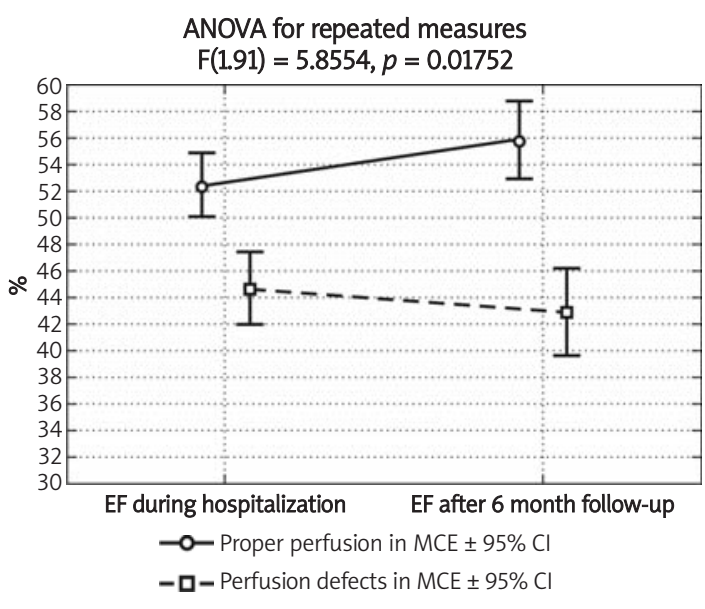

Figure 1. The ejection fraction during the index hospitalization and after 6-month follow-up in patients with perfusion defects on contrast echocardiography (dotted line, squares) or with proper perfusion (solid line, circles). Data are presented as average $\pm 95 \%$ confidence interval. ANOVA for repeated measures revealed statistically significant interaction between the presence of perfusion defects on echocardiography and change in ejection fraction during follow-up (F[1.91] $=5.8554$, $p=0.0175)$

Generally, the patients who had good MCE results also had, in the majority of cases, long-term improvement of ventricular function.

Patients from Group 2 had a higher incidence of death during the 6-month follow-up period. Two out of 39 patients (5\%) died, whereas there were no deaths in the group with good perfusion on MCE. Two patients (5\%) in Group 2 had non-fatal myocardial infarction, while there were no patients who sustained subsequent myocardial infarction in Group 1. The combined end-point (death and reinfarction) was significantly lower in patients without perfusion defect $(p=0.039)$.

\section{Discussion}

Our study showed that in patients with a first acute myocardial infarction with single vessel disease and successfully treated with primary $\mathrm{PCI}$, perfusion assessed by contrast echocardiography correlates well with certain clinical and echocardiographic parameters.

Recent studies have demonstrated that myocardial perfusion and metabolism are often abnormal, despite restoration of TIMI 3 flow in the infarct-related artery [1, 2, 4]. This "no-reflow" phenomenon is thought to be the result of microvascular obstruction [12, 13]. This phenomenon is a marker of more extensive myocardial tissue damage and has been proven to be associated with poorer functional recovery, an increased frequency of heart failure and poor survival $[4,14]$. That is why there is a growing need to establish a reliable marker of myocardial perfusion. Several markers of myocardial perfusion have been proposed. Among these are electrocardiographic (ST-segment elevation resolution), echocardiographic (MCE) and angiographic methods (TIMI myocardial perfusion grade - TMPG; myocardial blush grade - MBG; corrected TIMI frame count - CTFC) [15-18]. What all of them have in common is that they give information about perfusion and, at the same time, patients' prognosis. In the present study population despite TIMI 3 flow, more than $40 \%$ of patients had some degree of perfusion defect detected by MCE.

Myocardial contrast echocardiography, actively studied and compared with many diagnostic tools, appears to have feasibility and accuracy comparable with SPECT ([99m] Tc-sestamibi single photon emission computed tomography) imaging. Myocardial uptake using SPECT depends largely on myocardial microvascular volume. Myocardial contrast echocardiography detects not only microvascular volume but also blood flow [10, 19]. These differing mechanisms may affect the relative accuracies of MCE and SPECT for detecting myocardial viability and perfusion early after AMI.

Myocardial contrast echocardiography was also compared with SPECT early after first AMI [19]. Contractile reserve was assessed 3 months following revascularization. The sensitivity and specificity of MCE and SPECT were 83 and $78 \%$ ( $p=\mathrm{ns})$ and 78 and $45 \%(p=0.01)$, respectively. MCE was the only multivariate predictor of global recovery of function and contractile reserve $(\mathrm{OR}=3.5, p=0.01)$. The different physiological mechanisms employed by MCE and SPECT translate into different relative accuracies for the detection of myocardial perfusion and viability. Our findings showed that the presence of myocardial perfusion on MCE, from either collateral or antegrade flow, provided information on the extent of infarction and was associated with recovery of resting wall motion.

Our study compared simple-to-determine noninvasive markers of reperfusion. The principle findings were that: 1) MCE results also correlated with recovery of systolic left ventricular function and clinical outcome at 6-month follow-up, 2) anterior AMI was associated with a greater extent of perfusion defect in patients treated with primary $\mathrm{PCl}, 3$ ) treatment with a Ilb/IIla inhibitor was associated with better myocardial tissue perfusion in patients with AMI.

In our study serious adverse events (2 deaths and 2 non-fatal myocardial infarctions) occurred only in the group with perfusion defect on MCE.

Contrast echocardiography has previously been validated as the reference technique for evaluation of myocardial perfusion [1, 19-24]. The most 
important advantage is that it can be applied in the setting of acute myocardial infarction. The "noreflow" phenomenon is detected by contrast echocardiography in $16 \%$ of patients with TIMI 3 flow. Contrast echocardiography has been chosen by Agati et al. [25] as a tool to prove the superiority of primary $\mathrm{PCl}$ over thrombolytic therapy. Kamp et al. [9] carried out serial myocardial contrast echocardiography in patients after primary $\mathrm{PCl}$, showing that a significant reduction in size of the initial perfusion defect predicts functional recovery after 4 weeks, and these findings underscore the potential diagnostic value of the method. In AMI treated with PCl it may have not only therapeutic implications, but also prognostic significance, since "no-reflow" is associated with left ventricular dysfunction at follow-up [26].

We assume that our main division of the study population into one group with and one group without perfusion defects assessed by MCE was well founded and reasonable, as almost all echocardiographic and clinical outcomes turned out to be better in the group without perfusion defects on MCE.

Despite its potential to assess myocardial perfusion in a non-invasive way, MCE has not become a routine clinical tool thus far. An important limitation is that the underlying pathophysiology is complex. Moreover, appropriate interpretation of MCE requires experience and training. Furthermore, sophisticated software is needed to perform the studies optimally and to acquire accurate data.

As demonstrated in the present as well as in previous studies, reperfusion markers correlate with clinical outcome at follow-up.

In conclusion:

1) MCE results correlated with clinical outcome and recovery of systolic left ventricular function at 6-month follow-up,

2) anterior $\mathrm{AMI}$ was associated with a greater extent of perfusion defect in patients treated with primary $\mathrm{PCl}$,

3) treatment with a IIb/IIla inhibitor was associated with better myocardial tissue perfusion in patients with AMI,

4) MCE is an uncomplicated bedside method of perfusion assessment at a microvascular level in patients with $\mathrm{AMI}$ and gives additional information about actual infarct size, thus enabling early evaluation of the success of primary $\mathrm{PCl}$ results.

\section{Study limitations}

Regional wall thickening and motion were evaluated with echocardiography by semiquantitative analysis.

It was not possible to evaluate the exact role of various drugs, in particular ACE inhibitors, which were given in the majority of cases.
In our study, treatment with tirofiban was not randomized. Thirteen patients were treated with tirofiban at the discretion of the $\mathrm{PCl}$ operator. In those who received tirofiban, anterior myocardial infarction was prevalent. Nevertheless, patients treated with this IIb/IIla inhibitor had fewer perfusion defects with MCE than those who did not receive tirofiban.

The presented results cannot be applied to patients with complicated myocardial infarction or multivessel disease, who were excluded from the study.

\section{Acknowledgments}

The authors declare no conflict of interests.

\section{References}

1. Ito H, Tomooka T, Sakai N, et al. Lack of myocardial perfusion immediately after successful thrombolysis. A predictor of poor recovery of left ventricular function in anterior myocardial infarction. Circulation 1992; 85: 1699-705.

2. Wu KC, Zerhouni EA, Judd RM, et al. Prognostic significance of microvascular obstruction by magnetic resonance imaging in patients with acute myocardial infarction. Circulation 1998; 97: 765-72.

3. Kitazume H, Iwama T, Kubo I, Ageishi Y, Suzuki A. Noreflow phenomenon during percutaneous transluminal coronary angioplasty. Am Heart J 1988; 116: 211-5.

4. Roe MT, Ohman EM, Maas AC, et al. Shifting the openartery hypothesis downstream: the quest for optimal reperfusion. J Am Coll Cardiol 2001; 37: 9-18.

5. Lepper W, Hoffmann R, Kamp O, et al. Assessment of myocardial reperfusion by intravenous myocardial contrast echocardiography and coronary flow reserve after primary percutaneous transluminal coronary angioplasty [correction of angiography] in patients with acute myocardial infarction. Circulation 2000; 101: 2368-74. Erratum in: Circulation 2000;102: 482.

6. Morishima I, Sone T, Okumura K, et al. Angiographic noreflow phenomenon as a predictor of adverse long-term outcome in patients treated with percutaneous transluminal coronary angioplasty for first acute myocardial infarction. J Am Coll Cardiol 2000; 36: 1202-9.

7. Marzilli M, Gliozheni E, Marraccini P, Fedele S. Primary coronary angioplasty in acute myocardial infarction: Clinical correlates of the "no-reflow" phenomenon. Int J Cardiol 1998; 65 (suppl I): 23-8.

8. Schiller NB, Shah PM, Crawford M, et al. Recommendations for quantitation of the left ventricle by twodimensional echocardiography. American Society of Echocardiography Committee on Standards, Subcommittee on Quantitation of Two-Dimensional Echocardiograms. J Am Soc Echocardiogr 1989; 2: 358-67.

9. Kamp O, Lepper W, Vanoverschelde JL, et al. Serial evaluation of perfusion defects in patients with a first acute myocardial infarction referred for primary PTCA using intravenous myocardial contrast echocardiography. Eur Heart J 2001; 22: 1485-95.

10. Kaul S, Senior R, Dittrich H, Raval U, Khattar R, Lahiri A. Detection of coronary artery disease with myocardial contrast echocardiography. Circulation 1997; 96: 785-92. 
11. Judkins MP. Selective coronary arteriography. I. A percutaneous transfemoral technic. Radiology 1967; 89: 815-24.

12. Gerber BL, Rochitte CE, Melin JA, et al. Microvascular obstruction and left ventricular remodeling early after acute myocardial infarction. Circulation 2000; 101: 2734-41.

13. Michaels AD, Gibson CM, Barron HV. Microvascular dysfunction in acute myocardial infarction: focus on the roles of platelet and inflammatory mediators in the noreflow phenomenon. Am J Cardiol 2000; 85 (5A): 50B-60B.

14. Kunichika H, Ben-Yehuda O, Lafitte S, Kunichika N, Peters B, DeMaria AN. Effects of glycoprotein Ilb/Illa inhibition on microvascular flow after coronary reperfusion. A quantitative myocardial contrast echocardiography study. J Am Coll Cardiol 2004; 43: 276-83.

15. Gibson CM, Cannon CP, Murphy SA, Marble SJ, Barron HV, Braunwald E; TIMI Study Group. Relationship of the TIMI myocardial perfusion grades, flow grades, frame count, and percutaneous coronary intervention to long-term outcomes after thrombolytic administration in acute myocardial infarction. Circulation 2002; 105: 1909-13.

16. van't Hof AW, Liem A, Suryapranata H, Hoorntje JC, de Boer MJ, Zijlstra F. Angiographic assessment of myocardial reperfusion in patients treated with primary angioplasty for acute myocardial infarction: myocardial blush grade. Zwolle Myocardial Infarction Study Group. Circulation 1998; 97: 2302-6.

17. Gibson CM, Cannon CP, Daley WL, et al. TIMI frame count: a quantitative method of assessing coronary artery flow. Circulation 1996; 93: 879-88

18. Gibson CM, Cannon CP, Murphy SA, et al. Relationship of TIMI myocardial perfusion grade to mortality after administration of thrombolytic drugs. Circulation 2000; 101: 125-30.

19. Hickman M, Janardhanan R, Dwivedi G, Burden L, Senior R. Clinical significance of perfusion techniques utilizing different physiological mechanisms to detect myocardial viability: A comparative study with myocardial contrast echocardiography and single photon emission computed tomography. Int J Cardiol 2007; 114: 139-40.

20. Stone GW, Peterson MA, Lansky AJ, Dangas G, Mehran R, Leon MB. Impact of normalized myocardial perfusion after successful angioplasty in acute myocardial infarction. J Am Coll Cardiol 2002; 39: 591-7.

21. Haager PK, Christott P, Heussen N, Lepper W, Hanrath P, Hoffmann R. Prediction of clinical outcome after mechanical revascularisation in acute myocardial infarction by markers of myocardial reperfusion. J Am Coll Cardiol 2003; 41: 532-8.

22. Galiuto L, Iliceto S. Myocardial contrast echocardiography in the evaluation of viable myocardium after acute myocardial infarction. Am J Cardiol 1988; 81 (12A): 29G-32G.

23. Sakuma T, Otsuka M, Okimoto T, et al. Optimal time for predicting myocardial viability after successful primary angioplasty in acute myocardial infarction: a study using myocardial contrast echocardiography. Am J Cardiol 2001; 87: 687-92.

24. Kaul S. Myocardial contrast echocardiography: 15 years of research and development. Circulation 1997; 96 : 3745-60.

25. Agati L, Voci P, Bilotta F, et al. Influence of residual perfusion within the infarct zone on the natural history of left ventricular dysfunction after acute myocardial infarction: a myocardial contrast echocardiographic study. Am Coll Cardiol 1994; 24: 336-42.
26. Grines CL, Cox DA, Stone GW, et al. Coronary angioplasty with or without stent implantation for acute myocardial infarction. Stent Primary Angioplasty in Myocardial Infarction Study Group. N Engl J Med 1999; 341: 1949-56. 